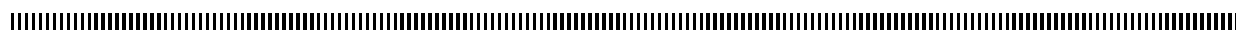
|

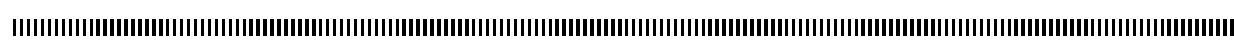
| |

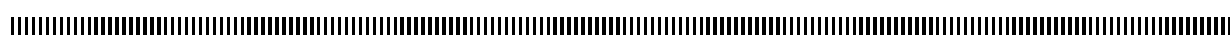
| |

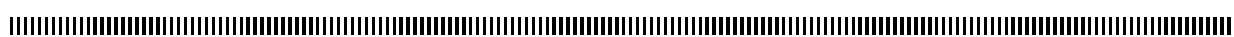

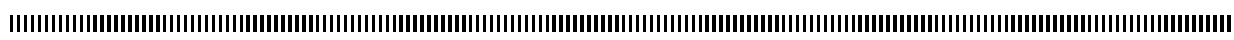

\title{
Décomposition de domaine pour un milieu poreux fracturé : un modèle en 3D avec fractures qui s'intersectent
}

\author{
Laila AMIR ${ }^{1,2}$, Michel KERN1, Vincent MARTIN ${ }^{1,3}$, Jean E ROBERTS ${ }^{1}$ \\ 1 INRIA-Rocquencourt, B.P. 105, F-78153 Le Chesnay Cedex \\ 2 ITASCA Consultants, S.A, 64, Chemin des Mouilles 69134 - ECULLY, France \\ 3 Université Pierre et Marie Curie, LJLL, B.P 187, 75252 Paris Cedex 05, France \\ Email: \{Laila.Amir, Michel.Kern, Vincent.Martin, Jean.Roberts $\} @$ inria.fr
}

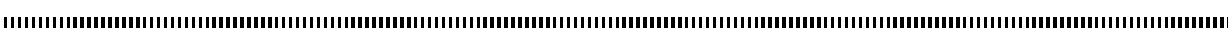

RÉSUMÉ. Dans cet article, nous nous intéressons à la modélisation de l'écoulement d'un fluide monophasique dans un milieu poreux faillé en utilisant les méhodes de décomposition de domaine. Le problème à résoudre est un problème d'interface non standard qui prend en compte l'écoulement dans les fractures. Dans l'approche proposée, la fracture est considée comme une interface active, les conditions de transmission et les échanges entre la roche et la fracture font intervenir les propriétés de l'écoulement dans la fracture.

ABSTRACT. In this paper, we are interested in modeling the flow of a single phase fluid in a porous medium with fractures, using domain decomposition methods. In the proposed approach, the fracture is regarded as an active interface, the transmission conditions and the exchanges between the rock and the fracture taking into account the flow in the fracture. The problem to be solved is then a non standard interface problem which takes into account the flow in the fractures.

MOTS-CLÉS : fractures, milieu poreux, décomposition de domaine, écoulement, interface, conditions de transmission

KEYWORDS : fractures, porous medium, domain decomposition, flux, interface, transmission conditions

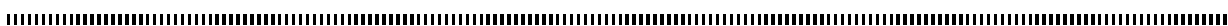

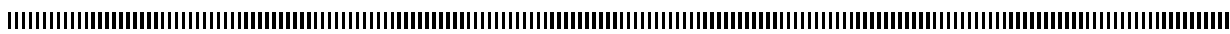

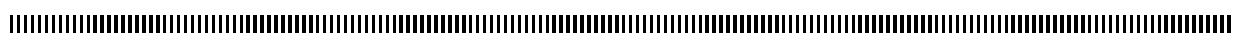

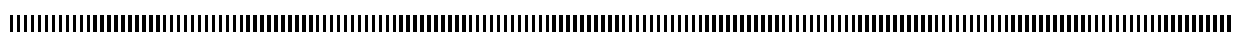
|

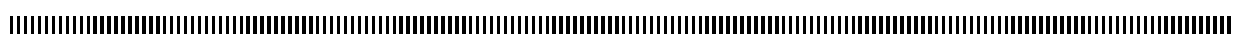
|

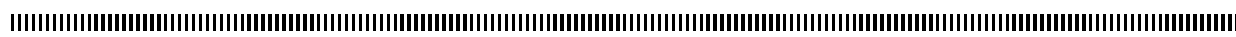

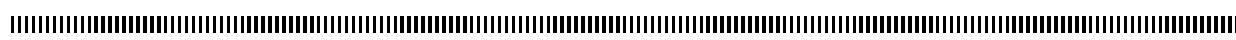




\section{Introduction}

Les milieux poreux sont généralement hétérogènes. L'une des raisons est la présence de fractures qui peuvent jouer un rôle hydraulique en contribuant de manière considérable à la capacité des sols à transporter l'eau et les polluants; ceci explique l'importance de la modélisation des fractures et de leur prise en compte lors d'une simulation d'écoulement dans un milieu poreux fracturé.

On peut distinguer deux types de fractures : les petites fractures qui apparaissent en grand nombre dans le milieu formant un réseau de fissures, dont les localisations précises sont difficiles à déterminer. Elles sont souvent prises en compte par un modèle de double porosité ([3], [4]) ou par une modélisation stochastique ([7], [8]). Le deuxième type de fracture est celui des grandes fractures qui représentent des discontinuités géologiques, et qui sont en général beaucoup plus perméables que le milieu ambiant, devenant des canaux privilégiés pour l'écoulement. Mais elle peuvent être au contraire peu perméables, et représentent alors des barrières géologiques. C'est le cas des grandes fractures qui nous intéresse ici.

Parmi les méthodes qui traitent le problème des grandes fractures dans un modèle numérique, les plus évidentes sont celles qui s'appuient sur le raffinement local du maillage au niveau de la fracture. Un modèle alternatif, présenté dans [1], [2], consiste à assimiler une fracture de grande perméabilité à une interface, et à traiter le problème résultant par une méthode de décomposition de domaine. Dans ce modèle, l'originalité repose sur le traitement de l'interface à travers laquelle la pression est continue mais le flux est discontinu. Ce modèle est généralisé au cas de fractures moins perméable dans [5], couplant sur l'interface sauts de pression et sauts de vitesse normale. Ces deux derniers modèles sont étudiés théoriquement pour le cas d'un domaine avec une seule fracture simple et numériquement avec des expériences en 2D. Les schémas numériques sont implémentés avec des techniques de décomposition de domaine. Cette article est la suite de ces travaux : ici nous nous intéressons au cas des fractures qui s'intersectent et nous introduisons un préconditionneur pour le problème de décomposition de domaine. Des expériences numériques en 3D sont effectuées.

\section{Le modèle}

Nous considérons l'écoulement d'un fluide incompressible et monophasique dans un milieu poreux $\Omega$ (ouvert connexe, régulier de $\mathbb{R}^{3}$ ). L'écoulement est gouverné par la loi de conservation de la masse et la loi de Darcy :

$$
\begin{aligned}
& \operatorname{div}(\vec{u})=f, \quad \text { dans } \Omega \\
& \vec{u}=-K \vec{\nabla} p, \quad \text { dans } \Omega,
\end{aligned}
$$

\section{Arima}


où $\vec{u}$ est la vitesse de Darcy, $p$ est la pression, $K$ est le tenseur de perméabilité et $f$ est un terme source. On suppose que le bord de $\Omega$ est décomposé en $\partial \Omega_{D}$ et $\partial \Omega_{N}$ où des conditions de Dirichlet, respectivement Neumann homogène, sont imposées :

$$
\begin{array}{ll}
p=p_{d} & \text { sur } \partial \Omega_{D} \\
\vec{u} \cdot \vec{\nu}=0 & \text { sur } \partial \Omega_{N},
\end{array}
$$

avec $\vec{\nu}$ est la normale unitaire sortante du bord $\partial \Omega$.

On suppose que $\Omega$ est divisé en plusieurs sous-domaines par des fractures. Les fractures sont aussi des milieux poreux, possédant leur propre perméabilités, mais leur dimension transverse est supposée plus petite de plusieurs ordres de grandeur que les deux autres, et elles sont assimilées à des interfaces (sans épaisseur, bien que l'épaisseur intervienne dans la loi de Darcy sur la fracture).

On prend comme problème modèle un domaine géologique $\Omega$, subdivisé en trois sousdomaines $\Omega_{i}, i=1,2,3$, naturellement séparés par des fractures $\gamma_{i, j}, i, j=1,2,3 ; i \neq$ $j$. La division en sous domaine est supposée être de telle sorte que

$$
\begin{gathered}
\bar{\Omega}=\bigcup_{i=1}^{3} \bar{\Omega}_{i}, \\
\Omega_{i} \cap \Omega_{j}=\emptyset \quad \text { et } \quad \bar{\Omega}_{i} \cap \bar{\Omega}_{j}=\bar{\gamma}_{i, j} \quad \text { pour tout } \quad i, j \in I=1,2,3,
\end{gathered}
$$

et on suppose que l'intersection des fractures est un segment $T$ :

$$
T=\bar{\gamma}_{12} \cap \bar{\gamma}_{23} \cap \bar{\gamma}_{31} \quad \text { et } \quad T=\bar{\gamma}_{12} \cap \bar{\gamma}_{23}=\bar{\gamma}_{23} \cap \bar{\gamma}_{31}=\bar{\gamma}_{31} \cap \bar{\gamma}_{12},
$$

de plus, $\quad \gamma_{i, j}=\left(\bar{\gamma}_{i, j} \cap \Omega\right) \backslash T$ pour tout $i, j \in I, i \neq j ; \quad$ et $\quad \gamma=\gamma_{12} \cup \gamma_{23} \cup \gamma_{31}$, voir Figure 1.

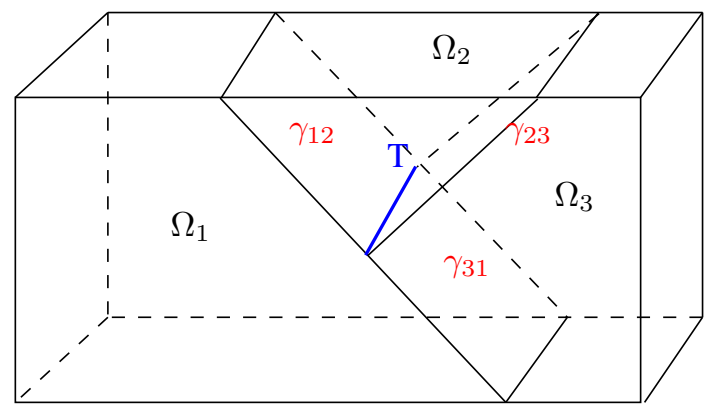

Figure 1. Géométrie du domaine de calcul 


\section{Arima -}

Sur chaque sous-domaine $\Omega_{i}$, on retrouve les équations d'écoulement usuelles (conservation de la masse et loi de Darcy):

$$
\begin{aligned}
\operatorname{div}\left(\vec{u}_{i}\right) & =f_{i} \text { dans } \Omega_{i} \\
\vec{u}_{i} & =-K_{i} \vec{\nabla} p_{i} \text { dans } \Omega_{i}
\end{aligned}
$$

où $p_{i} \vec{u}_{i}, f_{i}$ et $K_{i}$ sont respectivement la pression, la vitesse de Darcy, le terme source, et le tenseur de perméabilité dans $\Omega_{i}$, et avec la notation

$$
\Gamma_{D i}=\partial \Omega_{D} \cap \partial \Omega_{i} \quad \text { et } \quad \Gamma_{N i}=\partial \Omega_{N} \cap \partial \Omega_{i} \text { pour tout } i \in I,
$$

les conditions sur les bords extérieurs de $\Omega_{i}$ s'écrivent

$$
\begin{aligned}
p_{i} & =p_{d i} \text { sur } \Gamma_{D i} \\
\overrightarrow{u_{i}} \cdot \vec{\nu}_{i} & =0 \text { sur } \Gamma_{N i} .
\end{aligned}
$$

Sur les interfaces $\gamma_{i, j}, j \in I, j \neq i$, au lieu d'imposer la continuité de pression comme dans un problème de transmission standard, suivant [5] nous imposons

$$
p_{i}=p_{i, j}+\frac{d_{i, j}}{2 K_{\gamma_{i, j}}^{n}}\left(\xi \vec{u}_{i} \vec{\nu}_{i}-(1-\xi) \vec{u}_{j} \vec{\nu}_{j}\right) \quad \text { sur } \quad \gamma_{i, j},
$$

où $p_{i, j}$ est la pression dans la fracture $\gamma_{i, j}, K_{\gamma_{i, j}}^{n}$ est la perméabilité de la fracture dans la direction normale à celle-ci, $d_{i, j}$ est l'épaisseur de la fracture $\gamma_{i, j}$ et le paramètre $\xi$ permet d'obtenir différentes variantes.

REMARQUE. - L'équation (5) prend en compte un éventuel saut de pression à travers la fracture. Il est obtenu par une intégration dans l'épaisseur de la fracture, voir [5]. Notons que, si la fracture est très perméable (dans la direction normale à la fracture) c'est-à-dire si $K_{i, j}^{n}$ est suffisamment grand, le second terme du membre de droite de l'équation (5) sera négligeable devant les pressions, et il est possible de remplacer l'équation (5) par la continuité de la pression :

$$
p_{i}=p_{i, j}=p_{j} \text { sur } \gamma_{i, j} .
$$

C'est ce modèle qui a été étudié dans [1] pour le cas d'une fracture simple.

Pour la deuxième condition de transmission sur les interfaces $\gamma_{i, j}$, au lieu de la continuité du flux, on a, comme dans [1] et [5], un système d'équations qui modélise le flux dans les fractures, avec le saut de flux entre les sous domaine comme un terme source.

$$
\begin{aligned}
\operatorname{div}_{\gamma}\left(\vec{u}_{i, j}\right) & =f_{i, j}+\left(\vec{u}_{i} \cdot \vec{\nu}_{i}+\vec{u}_{j} \cdot \vec{\nu}_{j}\right) \text { sur } \gamma_{i, j} \\
\vec{u}_{i, j} & =-d_{i, j} K_{\gamma_{i, j}}^{\tau} \vec{\nabla}_{\gamma} p_{i, j} \quad \text { sur } \gamma_{i, j} \\
p_{i, j} & =p_{d \gamma_{i, j}} \quad \text { sur } \partial \gamma_{i, j} \cap \partial \Omega_{D} \\
\vec{u}_{i, j} \cdot \vec{\nu}_{i, j} & =0 \text { sur } \partial \gamma_{i, j} \cap \partial \Omega_{N},
\end{aligned}
$$

\section{Arima}


où $K_{\gamma_{i, j}}^{\tau}$ est la perméabilité tangentielle de la fracture, $p_{d \gamma_{i, j}}$ est la pression donnée sur son bord Dirichlet, et $\vec{\nu}_{i, j}$ est la normale extérieure à $\partial \gamma_{i, j}$. Les deux opérateurs $\operatorname{div}_{\gamma}$ et $\nabla_{\gamma}$ sont, respectivement, la divergence et le gradient surfaciques sur $\gamma$.

Sur le segment $T$, qui est lui-même une interface entre les fractures, on impose la continuité de pression et la continuité du flux :

$$
\begin{array}{r}
p_{i, j}=p_{T} \text { sur } T, \quad \forall i, j \in I ; i \neq j \\
\vec{u}_{12} \cdot \vec{\nu}_{12}+\vec{u}_{23} \cdot \vec{\nu}_{23}+\vec{u}_{31} \cdot \vec{\nu}_{31}=0 \text { sur } T,
\end{array}
$$

où $p_{T}$ est la pression sur $T$.

\section{Les formulations faible et discrète du problème global}

Dans la suite on adoptera la convention sur l'ensemble d'indice $I=\{1,2,3\}$, que $3+1=1$ (de sorte que les fractures sont toutes repérées par un couple $(i, i+1)$ ). Pour donner la formulation faible du problème globale on a besoin de deux espaces de Hilbert définis par:

$$
\begin{aligned}
& W=\left\{\quad u=\left(\vec{u}_{1}, \vec{u}_{2}, \vec{u}_{3}, \vec{u}_{12}, \vec{u}_{23}, \vec{u}_{31}\right): \vec{u}_{i} \in H\left(\operatorname{div}, \Omega_{i}\right),\right. \\
& \vec{u}_{i, i+1} \in H\left(\operatorname{div} \gamma, \gamma_{i, i+1}\right), \quad \vec{u}_{i} \cdot \vec{\nu}_{i}+\vec{u}_{i+1} \cdot \vec{\nu}_{i+1} \in L^{2}\left(\gamma_{i, i+1}\right) \forall i \in I, \\
& \text { et } \left.\sum_{i=1}^{3}\left(\vec{u}_{i+1} \cdot \vec{\nu}_{i i+1}\right)_{\mid T} \in L^{2}(T)\right\} \\
& M=\left\{p=\left(p_{1}, p_{2}, p_{3}, p_{12}, p_{23}, p_{31}, p_{T}\right):\right. \\
& \left.p_{i} \in L^{2}\left(\Omega_{i}\right), p_{i, i+1} \in L^{2}\left(\gamma_{i, i+1}\right), i \in I \text {, et } p_{T} \in L^{2}(T)\right\} \text {. }
\end{aligned}
$$

La norme sur $M$ est la norme du produit, alors que la norme sur $W$ est donnée par

$$
\begin{aligned}
\|u\|_{W}^{2}=\sum_{i \in I} & \left\|\vec{u}_{i}\right\|_{H\left(\operatorname{div}, \Omega_{i}\right)}^{2}+\sum_{i \in I}\left\|\vec{u}_{i, i+1}\right\|_{H\left(\operatorname{div}_{\gamma}, \gamma_{i, i+1}\right)}^{2} \\
& +\sum_{i \in I}\left\|\vec{u}_{i} \cdot \vec{\nu}_{i}+\vec{u}_{i+1} \cdot \vec{\nu}_{i+1}\right\|_{0, \gamma_{i, i+1}}^{2}+\left\|\sum_{i \in I} \vec{u}_{i, i+1} \cdot \vec{\nu}_{\gamma_{i, i+1}}\right\|_{0, T}^{2} .
\end{aligned}
$$

Soient $\alpha: W \times W \longrightarrow \mathbb{R}$ et $\beta: W \times M \longrightarrow \mathbb{R}$ les formes bilinéaires définies par

$$
\begin{aligned}
\alpha(u, v) & =\sum_{i \in I} \int_{\Omega_{i}} K_{i}^{-1} \vec{u}_{i} \cdot \vec{v}_{i}+\sum_{i \in I} \int_{\gamma_{i, i+1}}\left(d_{i, i+1} K_{\gamma_{i, i+1}}^{\tau}\right)^{-1} \vec{u}_{i, i+1} \cdot \vec{v}_{i, i+1} \\
& +\sum_{i \in I} \int_{\gamma_{i, i+1}} \frac{d_{i, i+1}}{2 K_{\gamma_{i, i+1}}^{n}}\left(\xi \vec{u}_{i} \cdot \vec{\nu}_{i}-(1-\xi) \vec{u}_{i+1} \cdot \vec{\nu}_{i+1}\right) \vec{v}_{i} \cdot \vec{\nu}_{i} \\
\beta(u, r)= & \sum_{i \in I} \int_{\Omega_{i}} \operatorname{div} \vec{u}_{i} r_{i}+\sum_{i \in I} \int_{\gamma_{i, i+1}}\left(\operatorname{div} \gamma_{i, i+1} \vec{u}_{i, i+1}-\left(\vec{u}_{i} \cdot \vec{\nu}_{i}+\vec{u}_{i+1} \cdot \vec{\nu}_{i+1}\right)\right) r_{\gamma_{i, i+1}} \\
& +\int_{T}\left(\sum_{i \in I} \vec{u}_{i, i+1} \cdot \vec{\nu}_{i, i+1}\right) r_{T},
\end{aligned}
$$

Arima 


\section{Arima -}

et soient les formes linéaires $L_{f}: M \longrightarrow \mathbb{R}$ et $L_{d}: W \longrightarrow \mathbb{R}$ définies par

$$
\begin{aligned}
L_{f}(r) & =\sum_{i \in I} \int_{\Omega_{i}} f_{i} r_{i}+\sum_{i \in I} \int_{\gamma_{i, i+1}} f_{i, i+1} r_{i, i+1} \\
L_{d}(v) & =\sum_{i \in I} \int_{\Gamma_{D i}} p_{d i} \vec{v}_{i} \cdot \vec{\nu}_{i}+\sum_{i \in I} \int_{\partial \gamma_{i, i+1} \cap \partial \Omega_{D}} p_{d \gamma_{i j}} \vec{v}_{i, i+1} \cdot \vec{\nu}_{i, i+1}
\end{aligned}
$$

Avec ces définitions des espaces et des formes, la formulation faible du problème (1) à (12) s'écrit

Trouver $(\vec{u}, p) \in W \times M$ tel que

$$
\begin{array}{lll}
(\mathcal{P}) \quad & =-L_{d}(\vec{v}), & \forall \vec{v} \in W \\
\beta(\vec{u}, \vec{v})-\beta(\vec{v}, p) & =L_{f}(r), & \forall r \in M .
\end{array}
$$

Sous les hypothèses que les perméabilités dans les sous domaines et dans les fractures soient bornées supérieurement et inférieurement par des constantes positives, et avec $\xi>$ $1 / 2$, l'existence et l'unicité de la solution de problème $(\mathcal{P})$ peuvent être démontrées par la théorie de Babuska-Brezzi.

Pour modéliser le champ de pression, et simuler correctement l'écoulement du fluide, on a discrétisé l'équation d'écoulement dans chaque sous-domaine $\Omega_{i}$ et dans chaque fracture $\gamma_{i, j}$ par la méthode des éléments finis mixtes, pour que le champ de vitesse soit calculé en même temps que le champ de pression, et que le schéma soit conservatif. Le domaine $\Omega$ est maillé de façon compatible avec la décomposition en sous-domaines $\Omega_{i}$ et les interfaces sont discrétisées de telle sorte que le maillage soit conforme au niveau de l'interface. L'intersection $T$ hérite d'une discrétisation des interfaces. Pour approcher le problème, on utilise les éléments de Raviart-Thomas de plus bas degré. On définit les espaces d'approximation $M_{h, i}, W_{h, i}, M_{h, \gamma_{i, j}}$ et $W_{h, \gamma_{i, i+1}}$ comme des sous-espaces respectivement de $L^{2}\left(\Omega_{i}\right), H\left(\operatorname{div}, \Omega_{i}\right), L^{2}\left(\gamma_{i, j}\right)$ et $H\left(\operatorname{div}_{\gamma}, \gamma_{i, j}\right)$ tel que $\left(M_{h, i}, W_{h, i}\right)$ est un espace de Raviart-Thomas de plus bas degré sur $\Omega_{i}$, et $\left(M_{h, \gamma_{i, j}}, W_{h, \gamma_{i, j}}\right)$ est un espace de Raviart-Thomas de plus bas degré sur $\gamma_{i, j}$. Soit $M_{h, T}$, le sous espace de $L^{2}(T)$ composé des fonctions constantes par intervalle de la discrétisation. Les espaces d'approximation $W_{h}$ et $M_{h}$, sous-espaces de dimension finie des espaces $W$ et $M$ sont définis par

$$
\begin{gathered}
W_{h}=\left(W_{h, 1} \times . . \times W_{h, 3} \times W_{h, \gamma_{12}} \times . . \times W_{h, \gamma_{31}}\right) \subset W \\
M_{h}=M_{h, 1} \times . . \times M_{h, 3} \times M_{h, \gamma_{12}} \times . . \times M_{h, \gamma_{31}} \times M_{h, T} \subset M .
\end{gathered}
$$

Le problème discret global s'écrit alors :

$$
\begin{array}{lll}
\text { Trouver }\left(\vec{u}_{h}, p_{h}\right) \in W_{h} \times M_{h} \text { tel que } & & \\
\left(\mathcal{P}_{h}\right) \quad \begin{array}{ll}
\alpha\left(\vec{u}_{h}, \vec{v}\right)-\beta\left(\vec{v}, p_{h}\right) \\
\beta\left(\vec{u}_{h}, r\right)
\end{array} & =-L_{d}(\vec{v}), & \forall \vec{v} \in W_{h} \\
& &
\end{array}
$$

\section{Arima}




\section{Décomposition de domaine pour le traitement des fractures}

Dans ce modèle, les fractures sont représentées par des interfaces entre les différentes parties du milieu poreux et il est naturel d'utiliser une méthode de décomposition de domaine pour résoudre le problème discrétisée. Pour cela, les inconnues $\vec{u}_{i}$, et $p_{i}$ à l'intérieur de chaque sous-domaine sont éliminées pour ramener le problème à un problème posé sur l'ensemble des interfaces, c.a.d. sur l'ensemble des fractures. Par simplicité, dans la suite de cette section, tous les problèmes formulés sont écrits sous forme forte. Évidemment nous avons travaillé avec la formulation faible et dans les espaces de discrétisation. Également par simplicité nous supposons que les conditions aux bords sur $\partial \gamma_{i, j} \cap \partial \Omega$ sont des conditions de Dirichlet.

Dans cette partie (et dans les expériences numériques de la section 5), nous travaillons avec le modèle de fractures très perméables, et nous avons donc remplacé l'équation (5) par l'équation (6) représentant la continuité de la pression à la traversée d'une fracture. Formellement, cela correspond à la limite $K_{\gamma_{i, i+1}}^{n} \rightarrow \infty$, bien que nous n'ayons pas encore justifié ce passage à la limite.

On considère le problème $\left(\mathcal{P}_{i}\right)$ dans le sous-domaine $\Omega_{i}$

$$
\begin{array}{llll} 
& \operatorname{div}\left(\vec{u}_{i}\right)=f_{i} & \text { dans } & \Omega_{i} \\
& \vec{u}_{i}=-K_{i} \vec{\nabla} p_{i} & \text { dans } & \Omega_{i} \\
\left(\mathcal{P}_{i}\right) & p_{i}=p_{d i} & \text { sur } & \Gamma_{D i} \\
& \vec{u}_{i} \cdot \vec{\nu}_{i}=0 & \text { sur } & \Gamma_{N i} \\
& p_{i}=p_{i, j} & \text { sur } & \gamma_{i, j}, \forall j \neq i .
\end{array}
$$

Par linéarité, la solution $\left(\vec{u}_{i}, p_{i}\right)$ peut s'écrire sous la forme :

$\vec{u}_{i}=\vec{u}_{i}^{0}+\vec{u}_{i}^{*}$ et $p_{i}=p_{i}^{0}+p_{i}^{*}$ avec $\left(\vec{u}_{i}^{0}, p_{i}^{0}\right)$ et $\left(\vec{u}_{i}^{*}, p_{i}^{*}\right)$ les solutions respectives de

$$
\begin{aligned}
& \operatorname{div}\left(\vec{u}_{i}^{0}\right)=0 \quad \text { dans } \quad \Omega_{i} \quad \operatorname{div}\left(\vec{u}_{i}^{*}\right)=f_{i} \quad \text { dans } \quad \Omega_{i}
\end{aligned}
$$

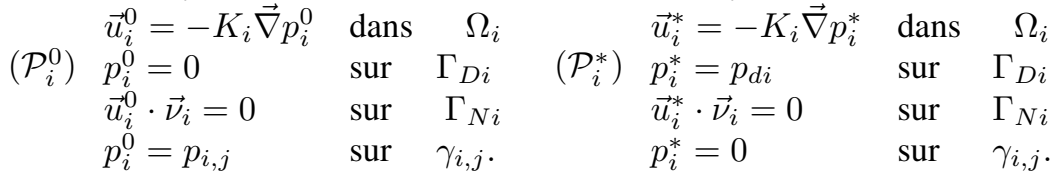

Les équations (7),..,(11) sur l'interface $\gamma_{i, j}$ peuvent s'écrire

$$
\begin{array}{lll}
\operatorname{div}_{\gamma} \vec{u}_{i, j}=f_{\gamma_{i, j}}+\left(\vec{u}_{i}^{*} \cdot \vec{\nu}_{i}+\vec{u}_{j}^{*} \cdot \vec{\nu}_{j}\right)+\left(\vec{u}_{i}^{0} \cdot \vec{\nu}_{i}+\vec{u}_{j}^{0} \cdot \vec{\nu}_{j}\right) & \operatorname{sur} \gamma_{i, j} \\
\left(\mathcal{P}_{\gamma_{i, j}}\right) & \vec{u}_{i, j}=-d_{i, j}, K_{\gamma_{i, j}}^{\tau} \vec{\nabla}_{\gamma} p_{i, j} & \operatorname{sur} \gamma_{i, j} \\
& p_{i, j}=p_{d \gamma_{i, j}} & \operatorname{sur} \partial \gamma_{i, j} \cap \partial \Omega \\
& p_{i, j}=p_{T} & \operatorname{sur} T .
\end{array}
$$




\section{Arima -}

En éliminant l'inconnue $\vec{u}_{i, j}$, les deux premières équations de ce système peuvent être remplacées par l'équation

$$
\operatorname{div}_{\gamma}\left(-d_{i, j}, K_{\gamma_{i, j}}^{\tau} \vec{\nabla}_{\gamma} p_{i, j}\right)=f_{\gamma_{i, j}}+\left(\vec{u}_{i}^{*} \cdot \vec{\nu}_{i}+\vec{u}_{j}^{*} \cdot \vec{\nu}_{j}\right)+\left(\vec{u}_{i}^{0} \cdot \vec{\nu}_{i}+\vec{u}_{j}^{0} \cdot \vec{\nu}_{j}\right) \quad \operatorname{sur} \gamma_{i, j} .
$$

(Dans la formulation discrète par éléments finis mixtes ceci correspond à l'utilisation d'une règle de quadrature pour la matrice de masse pondérée pour l'espace $W_{h, \gamma_{i, j}}$, pour obtenir une matrice diagonale rendant l'élimination des inconnues dans $W_{h, \gamma_{i, j}}$ facile)

Pour ramener le problème à un problème posé sur l'interface, on définit l'espace

$$
M_{f}=L^{2}(\gamma) \times L^{2}(T)=L^{2}\left(\gamma_{12}\right) \times L^{2}\left(\gamma_{23}\right) \times L^{2}\left(\gamma_{31}\right) \times L^{2}(T)
$$

Alors $\lambda \in M_{f}$ s'écrit : $\lambda=\left(\lambda_{\gamma}, \lambda_{T}\right)=\left(\lambda_{12}, \lambda_{23}, \lambda_{31}, \lambda_{T}\right)$

et on écrit $\lambda_{i}$ pour $\left(\lambda_{i-1, i}, \lambda_{i, i+1}\right)=\lambda_{\gamma \mid \partial \Omega_{i} \cap \gamma}, i \in I$. On écrira aussi $\gamma_{i}$ pour $\partial \Omega_{i} \cap \gamma=$ $\cup_{j \in I, j \neq i} \gamma_{i, j}$.

Pour chaque sous-domaine $\Omega_{i}$, l'opérateur de Steklov-Poincaré $\mathcal{S}_{i}$ est défini de façon que, pour $\lambda \in M_{f}$,

$$
\mathcal{S}_{i}(\lambda)=\mathcal{S}_{i}\left(\lambda_{i}\right)=-\left(\vec{u}_{i}^{0} \cdot \vec{\nu}_{i}\right)_{\mid \gamma_{i}},
$$

où $\left(\vec{u}_{i}^{0}, p_{i}^{0}\right)$ est la solution du problème $\left(\mathcal{P}_{i}^{0}\right)$ avec $p_{i, j}=\lambda_{i, j}, \forall j \neq i$, et l'opérateur $\chi_{i}$ est défini par :

$$
\chi_{i}\left(f_{i}, p_{d i}\right)=\left(\vec{u}_{i}^{*} \cdot \vec{\nu}_{i}\right)_{\mid \gamma_{i}},
$$

où $\left(\vec{u}_{i}^{*}, p_{i}^{*}\right)$ est la solution du problème $\left(\mathcal{P}_{i}^{*}\right)$.

En prolongeant $\mathcal{S}_{i}(\lambda)$ et $\chi_{i}$ à toute $\gamma$ avec une extension par 0 sur $\gamma \backslash \gamma_{i}$, on peut définir

$$
\mathcal{S}(\lambda)=\sum_{I} \mathcal{S}_{i}(\lambda) \quad \text { et } \quad \chi\left(f, p_{d}\right)=\sum_{I} \chi_{i}\left(f_{i}, p_{d i}\right) .
$$

Alors on peut écrire le système sur l'interface globale

$$
\begin{aligned}
\mathcal{S}(\lambda)-\operatorname{div}_{\gamma}\left(d_{\gamma} K_{\gamma}^{\tau} \vec{\nabla}_{\gamma} \lambda\right) & =f_{\gamma}+\chi\left(f, p_{d}\right) & & \text { sur } \gamma \\
\lambda & =p_{d \gamma} & & \text { sur } \partial \gamma \cap \partial \Omega \\
\left(\mathcal{P}_{\gamma}^{*}\right) & & & \operatorname{sur} T, i \in I \\
\lambda_{i, i+1}-\lambda_{T} & =0 & & \operatorname{sur} T,
\end{aligned}
$$

où $d_{\gamma}, K_{\gamma}^{\tau}$ et $f_{\gamma}$ sont définies de façon évidente, et l'opérateur $\mathcal{S}_{T}$ est défini par

$$
\mathcal{S}_{T}(\lambda)=\sum_{i \in I}\left(-d_{i, i+1} K_{\gamma_{i, i+1}}^{\tau} \vec{\nabla}_{\gamma} \lambda_{i, i+1}\right)_{\mid T} \cdot \nu_{i, i+1}
$$

pour que la dernière équation du système global représente la continuité du flux imposé sur T dans l'équation (12).

\section{Arima}


REMARQUe. - Dans ce problème les équations sur les interfaces possèdent un terme supplémentaire (le terme $\left.-\operatorname{div}_{\gamma}\left(d K_{\gamma} \vec{\nabla}_{\gamma} \lambda_{\gamma}\right)\right)$ par rapport à ceux que l'on obtient en résolvant un problème global par décomposition de domaines en l'absence de fracture. Ce terme modélise l'écoulement dans les fractures.

Ce système est symétrique et défini positif, et nous proposons donc le résoudre par une méthode itérative de gradient conjugué. Chaque itération demande la résolution d'un problème de Dirichlet dans chaque sous-domaine, ainsi qu'un calcul d'écoulement dans la fracture.

Pour accélérer la convergence de la méthode itérative, il est essentiel d'introduire un préconditionneur. Dans les problèmes sans fractures, pour la méthode de décomposition de domaine classique, le préconditionneur de Neumann-Neumann a prouvé son efficacité [6]. Il revient à inverser chacun des opérateurs $S_{i}$ localement, et il demande la résolution d'un problème de Neumann sur chaque sous-domaine. Par contre, en comparant les ordres différentiels des différents opérateurs du système sur l'interface globale, il est naturel de prendre comme préconditionneur l'inverse de l'opérateur d'écoulement sur la fracture, qui est d'ordre 2, alors que les opérateurs de Steklov-Poincaré sont seulement d'ordre 1. En fonction de la grandeur de la conductivité $K_{\gamma}^{\tau}$, l'un ou l'autre de ces préconditionneurs, ou une combinaison pourrait se révéler efficace. Nous prévoyons d'explorer cet aspect dans un travail futur.

\section{Résultats numériques}

\subsection{Description des cas tests}

Dans ce paragraphe nous présentons quelques résultats numériques pour illustrer les propriétés du modèle présenté dans cet article. Le modèle a été étudié à partir d'un jeu de données, et d'un ensemble de tests qui a permis d'avoir un premier aperçu de la performance de l'algorithme de décomposition de domaine avec fractures.

Pour les expériences numériques, on a considéré un parallélépipède rectangle $\Omega$, de dimension $10 \mathrm{~m} \times 2 \mathrm{~m} \times 5 \mathrm{~m}$, qui contient en plus des trois fractures du problème modèle (Figure 1), une fracture simple isolée (Figure 2).

Trois cas tests sont considérés. Dans les trois cas, une pression égale à 1 sur les extrémités supérieures des fractures et une pression égale à 0 sur les extrémités inférieures des fractures ont été imposés. Dans tout le domaine, la perméabilité est égale à 1, sauf sur les fractures, où la perméabilité (transverse et horizontal) est égale à $10^{4} \mathrm{~m} \cdot \mathrm{s}^{-1}$ et l'épaisseur à $10^{-2} \mathrm{~m}$ (la perméabilité transverse dans la fracture multipliée par son épaisseur est égale à $100 \mathrm{~m}^{2} \cdot \mathrm{s}^{-1}$ et l'épaisseur divisée par la perméabilité horizontal est égale à $10^{-6} \mathrm{~s}$ ). 


\section{Arima -}

\subsection{1. Écoulement horizontal}

Pour le premier test un gradient de pression horizontal est imposé. La figure 2 représente la géométrie et les conditions aux limites, pour cet exemple : on impose $p=1$ sur le bord latéral gauche et $p=0$ sur le bord latéral droit et une condition de flux nul sur le reste du bord.

$\vec{u} \cdot \vec{n}=0$

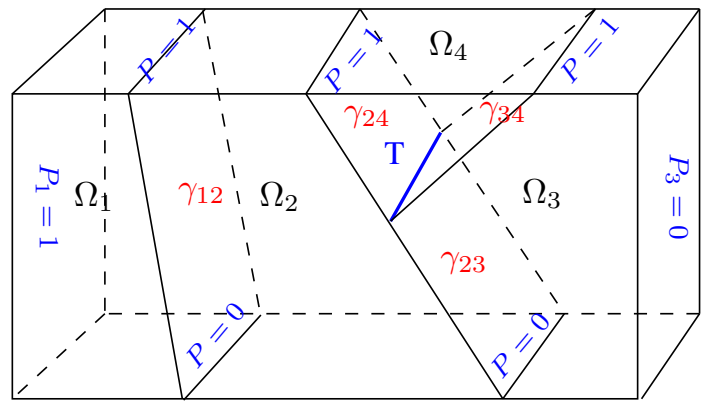

$\vec{u} \cdot \vec{n}=0$

Figure 2. Géométrie et conditions aux limites du premier cas test

La figure 3 présente le champ de pression en l'absence de fissures (Chaque sousdomaine est représenté séparément). Comme on peut s'y attendre, l'écoulement est uniquement horizontal, la pression varie linéairement de $p=1$ en $x=0$ à $p=0$ en $x=10$.

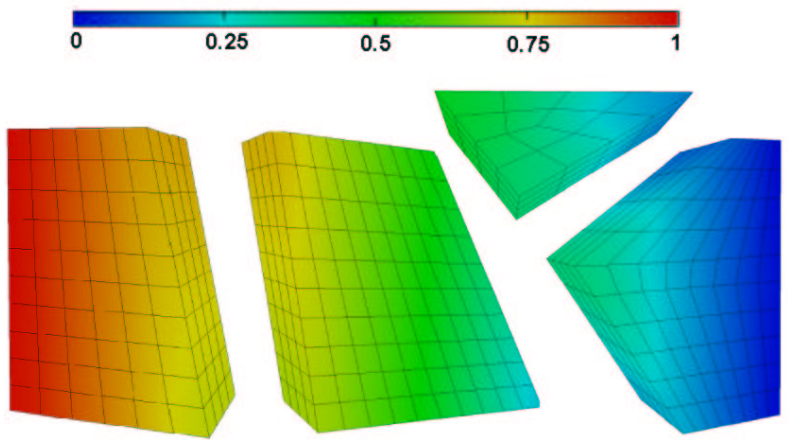

Figure 3. La pression donnée par le modèle sans fractures : $K_{i}=1, \quad i=1, . ., 4$.

Si l'on prend en compte les fractures, la situation change, comme le montre la figure 4 : l'effet d'écoulement dans les fractures est visible. En plus de l'écoulement horizontal il y a un écoulement le long des fractures.

\section{Arima}




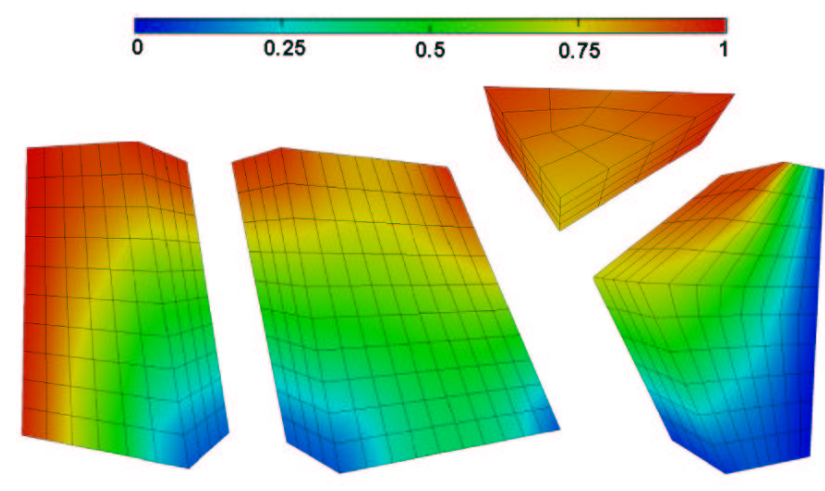

Figure 4. La pression donnée par le modèle avec fractures : $d K_{\gamma}=100, K_{i}=1, i=$ $1, . ., 4$.

\subsection{2. Écoulement du aux fractures}

Dans le deuxième cas test, la pression est égale à 1 sur les deux bords latéraux de $\Omega$. En l'absence de fractures la pression serait constante (nous ne la représentons pas), par contre la figure 5 montre l'effet du aux fractures. En comparant les figures 4 et 5, on voit également la différence due à la condition aux limites imposé sur le bord droit.

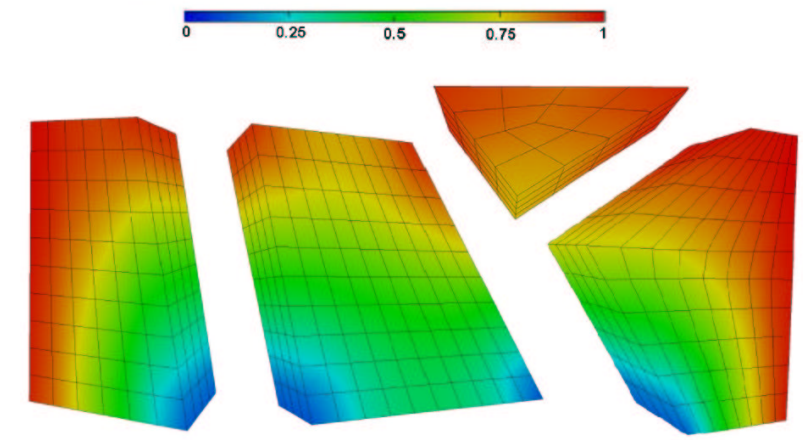

Figure 5. La pression donnée par le modèle avec fractures : $d K_{\gamma}=100, K_{i}=1, i=$ $1, . ., 4$.

\subsection{3. Écoulement vertical}

Pour le troisième test un gradient de pression vertical à été imposé. La figure 6 représente la géométrie et les conditions aux limites pour cet exemple : on a $p_{i}=1$ sur le bord supérieur de chaque sous-domaine et $p_{i}=0$ sur le bord inférieur et une condition de flux nul sur le reste du bord. 
Arima -

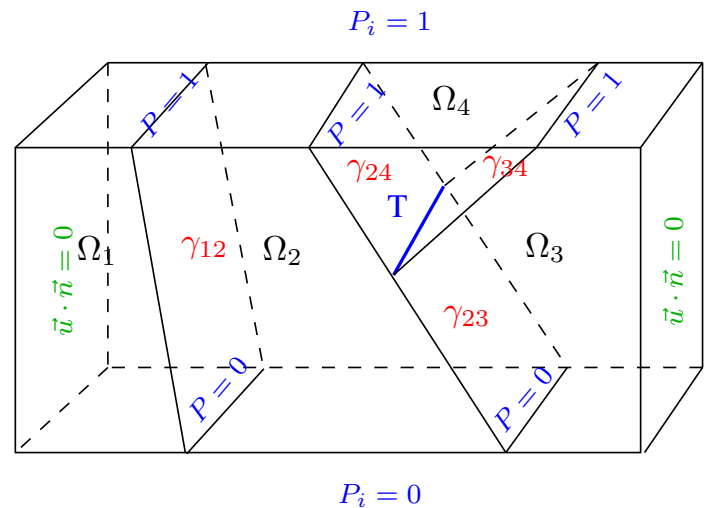

Figure 6. Géométrie et conditions aux limites du troisième cas test

La figure 7 présente le champ de pression en l'absence de fractures. On voit que la pression diminue linéairement du haut au bas d'une valeur égale à 1 à une égale à 0.

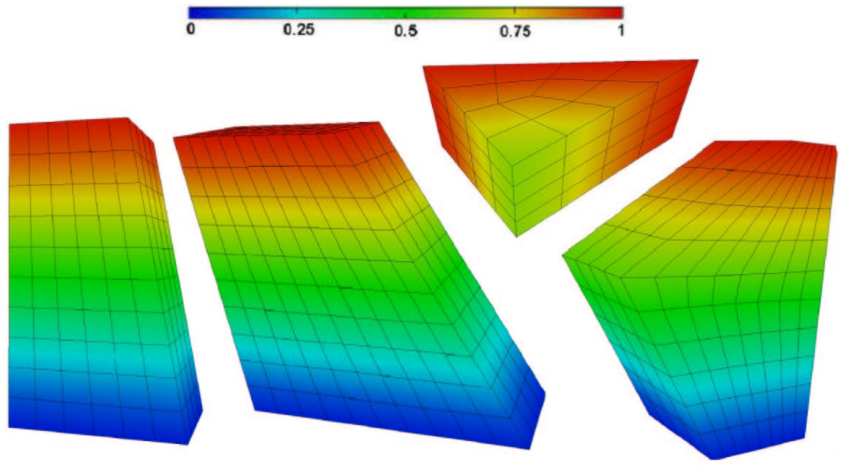

Figure 7. La pression donnée par le modèle sans fractures : $K_{i}=1, \quad i=1, . ., 4$.

En comparant ce résultat avec celui donné en présence des fractures (figure 8), on constate en particulier l'effet produit par les 3 fractures qui s'intersectent. L'écoulement est, en effet, plus important au voisinage de l'intersection, comme cela se voit en particulier sur $\Omega_{4}$.

Arima 


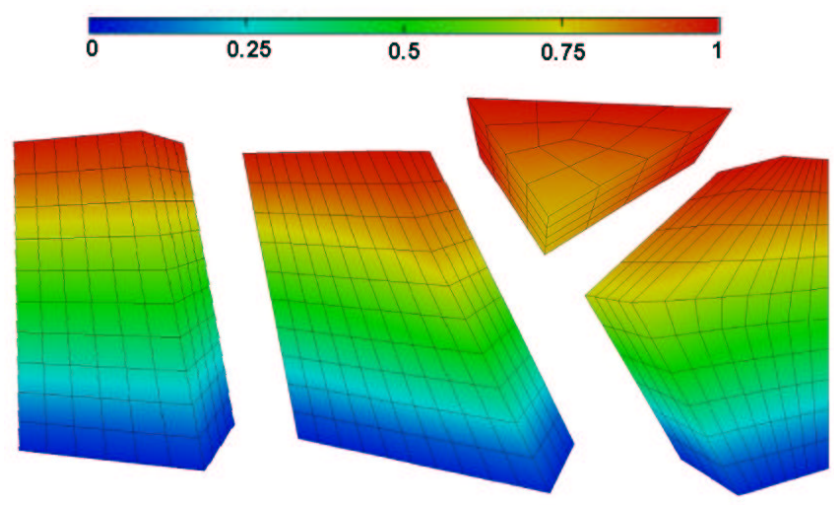

Figure 8. La pression donnée par le modèle avec fractures : $d K_{\gamma}=100, K_{i}=1$.

\subsection{Performance du préconditionneur}

Pour mettre en évidence l'importance du préconditionnement, l'algorithme du gradient conjugué a été utilisé sans préconditionnement, puis en préconditionnant par l'inverse de l'opérateur d'écoulement dans les fractures. On a demandé une réduction relative de $10^{-8}$ pour le résidu du gradient conjugué. La figure 9 , où le logarithme du résidu est tracé en fonction du nombre d'itérations, présente les résultats obtenus.

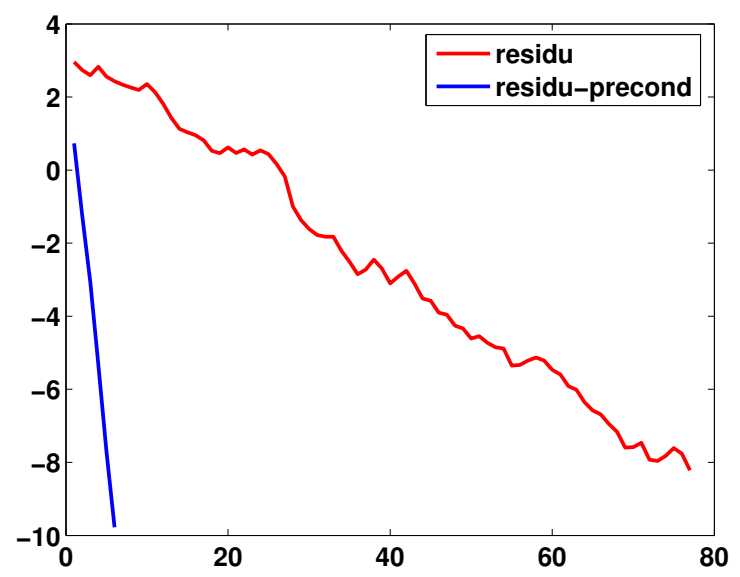

Figure 9. Test 1 : l'efficacité du préconditionneur 


\section{Arima}

Sans préconditionnement la méthode du gradient conjugué converge lentement, mais avec préconditionnement, on remarque une amélioration importante de la convergence, puisque le nombre d'itérations à la convergence est alors divisé par 10. De plus, la courbe de convergence de l'algorithme préconditionné est plus régulière que celle de l'algorithme sans préconditionnement. Le préconditionneur a un coût faible : On résout un problème 2D sur le réseau des fractures et la matrice peut être factorisée une fois pour toutes.

Pour mettre en évidence l'influence du coefficient $d K_{\gamma}$, nous comparons sur la figure 10 les courbes de convergence de l'algorithme pour différentes valeurs de $d K_{\gamma}$. On constate bien que le préconditionnement est plus efficace quand $d K_{\gamma}$ augmente.

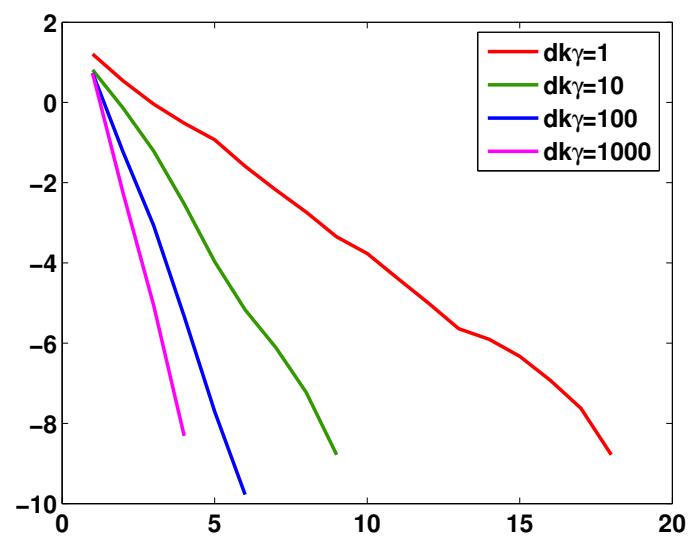

Figure 10. Test 2 : l'efficacité du préconditionneur en fonction de $d K_{\gamma}$

Pour obtenir un préconditionnement efficace pour les petites valeurs de $d K_{\gamma}$ (le cas où les fractures représentent des barrières), on peut envisager d'utiliser une combinaison linéaire du préconditionneur Neumann-Neumann et l'inverse de l'opérateur d'écoulement dans les fractures.

\section{Conclusion}

On a présenté dans cet article un modèle d'écoulement dans un milieu poreux traversé par des grandes fractures qui peuvent éventuellement se couper. Dans ce modèle, les fractures sont assimilées à des interfaces à travers lesquelles ni la pression ni le flux ne sont supposés être continus. Nous avons fait des expériences numériques dans un domaine $3 \mathrm{D}$ pour le cas où la perméabilité dans les fractures est suffisamment élevée pour que la pression soit considérée continue à travers les fractures. Une méthode de décom-

\section{Arima}


position de domaine a été utilisée afin de se ramener à un problème posé sur l'ensemble des interfaces. Un préconditionneur pour le problème algébrique a été introduit.

Nous envisageons deux suites à ce travail : l'extension au cas du transport de solutés et l'extension de la formulation par décomposition de domaine aux cas de fractures peu perméables qui se coupent. Nous poursuivrons aussi nos études sur le préconditionneur par une étude théorique.

\section{Bibliographie}

[1] C. Alboin, J. Jaffré et J. E. Roberts. - Domain decomposition for flow in fractured porous media. - In : Domain Decomposition Methods in Sciences and Engineering, Domain Decomposition Press, pp. 365-373, - Bergen, 1999.

[2] C. Alboin and J. Jaffré and J. E. Roberts and C.Serres, - Modeling Fractures as Interfaces for Flow and Transport in Porous Media, - Contemporary Mathematics, Volume 295, pp. 13-24, 2002

[3] J. Douglas Jr. et T. Arbogast - Dual porosity models for flow in naturally fractured reservoirs, Dynamics of Fluids in Hierarchial Porous Formations, J. H. Cushman (ed.), Academic Press, 1990, pp. 177-221.

[4] J. Douglas Jr, T. Arbogast et U. Hornung- Derivation of the double porosity model of single phase flow via homogenization theory, SIAM J. Math. Anal., (1990) 21 823-836.

[5] V. Martin, J. Jaffré, J. E. Roberts - Modeling fractures and barriers as interfaces for flow in porous media. SIAM Journal on Scientific Computing, vol. 26, No 5, pp. 1667-1691 (electronic), 2005.

[6] P. Le Tallec -Domain decomposition methods in computational mechanics. Computational Mechanics Advances, vol. 1, No 2, pp. 122-220, February 1994.

[7] J-R. de Dreuzy and J. Erhel- Efficient algorithms for the determination of the connected fracture network and the solution to the steady-state flow equation in fracture networks. Computers and Geosciences, Volume 29, pp. 107-111, 2003.

[8] J-R. de Dreuzy, P. Davy, J. Erhel and J. de Bremond d'Ars - Anomalous diffusion exponents in continuous two-dimensional multifractal media. / Physical Review E. /, Volume 70, 016306 (6 pages), 2004 